\title{
MODELAGEM DA BIOMASSA TOTAL E DA LENHA POR UNIDADE DE ÁREA PARA BRACATINGAIS NATIVOS ${ }^{1}$
}

\author{
Ronan Felipe de Souza², Sebastião do Amaral Machado ${ }^{3}$ e Afonso Figueiredo Filho ${ }^{4}$
}

RESUMO - A bracatinga (Mimosa scabrella Benth.) é uma espécie pioneira nativa da Floresta Ombrófila Mista de rápido crescimento e cultivada, predominantemente, na forma de povoamentos puros ou consorciada com culturas agrícolas pelos pequenos e médios produtores da região metropolitana de Curitiba. O objetivo deste estudo foi selecionar equações estimativas da biomassa total e da lenha de bracatingais por unidade de área nas diferentes idades, disponibilizando, assim, uma ferramenta simples e de fácil aplicação durante o processo de comercialização dos povoamentos. Os dados são provenientes de 272 parcelas temporárias com idades variando de 3 a 18 anos. Foram testados 21 modelos tradicionais: 10 aritméticos e semilogarítmicos e 11 logarítmicos. Foram também desenvolvidas equações pelo processo Stepwise a partir de uma matriz de correlação. As equações ajustadas foram comparadas pelo Coeficiente de determinação ajustado $\left(\mathrm{R}_{\mathrm{aj}}^{2}\right)$, Erro-padrão da estimativa percentual $\left(\mathrm{S}_{\mathrm{yx}} \%\right)$, teste $\mathrm{F}$ e Distribuição gráfica de resíduos. Duas equações para cada caso foram selecionadas, sendo uma tradicional e a outra desenvolvida por Stepwise. Para a biomassa total, foram selecionadas a equação de Clutter e a equação aritmética desenvolvida por Stepwise; e para a biomassa da lenha, a equação da variável combinada, proposta por Spurr, e a equação aritmética de Stepwise. Por fim, realizou-se teste Qui-quadrado $\left(x^{2}\right)$ a partir de uma amostra de 17 parcelas extraídas da base de dados, em que as quatro equações selecionadas foram consideradas válidas para a estimativa da biomassa total e da lenha dos bracatingais da região metropolitana de Curitiba.

Palavras-chave: Bracatinga; Modelos estimativos; Teste de validação.

\section{MODELLING OF TOTAL AND FIREWOOD BIOMASS BY UNNIT OF AREA FOR NATIVE Mimosa scabrella Benth. STANDS}

\begin{abstract}
Mimosa scabrella Benth. is a rapid-growth native pioneer specie from the Ombrophylous Mix Forest biome. It is cultivated predominantly in pure stand form or intercropped with agricultural crops by small and medium producers in the metropolitan region of Curitiba. The objective this study was to select estimative models for total and firewood biomass of M. scabrella stands per unit of area at different ages. It was data were collected from 272 temporary samples plots aged 3 to 18 years old. We tested 21 traditional models: 10 arithmetic and semi-logarithmic and 11 logarithmic; it was also developed equations by the Stepwise process from a correlation matrix. The adjusted equation were compared basing on $R_{a j}^{2}, S_{y x} \%, F$ value and on graphic analysis of residuals. Two equations for each case were selected, being one traditional and other developed by Stepwise. The Clutter equation and arithmetic developed by Stepwise were selected for total biomass. For firewood, were selected the combined variable equation proposed by Spurr, and the arithmetic equation developed by Stepwise. The validation of equations was performed by Chi-square test from 17 plots randomly selected from the data base, in which four equations were considered valid to estimate total and firewood biomass for M. scabrella stands in the metropolitan region of Curitiba.
\end{abstract}

Keyworks: Mimosa scabrella; Estimative models; Validation test.

\footnotetext{
${ }^{1}$ Recebido em 25.08.2010 aceito para publicação em 07.11.2013.

${ }^{2}$ Programa de Pós-graduação em Engenharia Florestal, Universidade Federal do Paraná. E-mail:<ronanflorestal@gmail.com>

${ }^{3}$ Departamento de Ciências Florestais da Universidade Federal do Paraná, Paraná, Brasil. E-mail:<samachado@ufpr.br>

${ }^{4}$ Universidade Estadual do Centro-Oeste, UNICENTRO, Brasil. E-mail:<afig@floresta.ufpr.br>.
} 


\section{INTRODUÇÃO}

A bracatinga (Mimosa scabrella Benth) é uma espécie pioneira, heliófila, nativa da Floresta Ombrófila Mista, com dominância de Araucaria angustifolia. Em 1909, Romário Martins iniciou uma campanha de divulgação para estabelecer povoamentos de bracatinga com fins de produção de lenha na região metropolitana de Curitiba, devido ao rápido crescimento e à simplicidade de práticas culturais necessárias para o plantio e manutenção desta espécie (CARPANEZZI et al., 1997).

Laurent et al. (1990) estimaram a área plantada de bracatinga na região metropolitana de Curitiba em 60.000 ha. Atualmente, a área plantada com essa espécie tem diminuído, embora o sistema tradicional de cultivo ainda seja atividade de grande importância econômica para pequenos e médios produtores da região. Dessa forma, estudos que permitem o avanço das técnicas silviculturais e de manejo da espécie, bem como a estimativa acurada da produção, são de grande importância, contribuindo para a ampliação do retorno financeiro aos produtores.

Além da comercialização na forma do volume de madeira em metros cúbicos $\left(\mathrm{m}^{3}\right)$, os bracatingais também são muito comercializados pelo peso de matéria seca dos compartimentos passíveis de utilização na forma de energia (lenha, carvão). Assim, a estimativa acurada da biomassa total e da lenha por unidade de área é de grande valia, permitindo a quantificação dos valores referentes à madeira que será comercializada, bem como valores de biomassa seca remanescente nos povoamentos que será incorporada ao solo.

Oliveira et al. (2005) desenvolveram equações estimativas do volume por unidade de área para fragmentos de floresta natural da região de Viçosa, Minas Gerais. Em seus resultados, esses autores destacaram a alta correlação do volume com as variáveis independentes área basal por ha $(\mathrm{G})$, altura total média $(\overline{\mathrm{h}})$ e altura dominante $\left(\mathrm{h}_{\mathrm{dom}}\right)$, o que resultou na seleção de equações que envolveram essas variáveis como as mais acuradas. Analisando o efeito da inclusão da altura do fuste na forma logaritmizada, esses mesmos autores encontraram resultados satisfatórios, observando valores de coeficiente de determinação superiores a 0,95 para as equações.

Machado et al. (2008) desenvolveram equações estimativas para o volume por unidade de área para bracatingais da região metropolitana de Curitiba. Os resultados revelaram alta correlação das variáveis combinadas $\mathrm{G} \overline{\mathrm{h}}$ e $\mathrm{Gh}_{\mathrm{dom}}$ com o volume total e da lenha nas formas aritmética e logarítmica, sendo estas as variáveis incluídas na equação Stepwise, desenvolvida pelos referidos autores. Dos modelos aritméticos e semilogarítmicos selecionados da literatura, os que apresentaram melhores ajustes foram aqueles que envolveram a variável $\mathrm{G}$, sendo considerados inadequados os que não compunham essa variável. As equações logarítmicas apresentaram melhores ajustes do que as aritméticas, sempre exibindo em sua estrutura as variáveis $G$ e $G h_{\text {dom }}$ na forma logaritmizada.

Objetivando selecionar equações capazes de estimar com precisão a biomassa total e da lenha por unidade de área nas diferentes idades dos bracatingais nativos e manejados de forma tradicional da região metropolitana de Curitiba, foram ajustados e comparados modelos tradicionais aritméticos, semilogarítmicos e logarítmicos. Também foram desenvolvidas e testadas equações pelo processo Stepwise a partir de uma matriz de correlação linear simples.

\section{MATERIAL E MÉTODOS}

\subsection{Caracterização da área de estudo}

No total foram incluídos 14 municípios, abrangendo uma área de influência de 731.850 ha. Machado et al. (2008) destacaram que esta área de estudo está compreendida entre Tunas do Paraná como a localidade mais ao Norte e Agudos do Sul no extremo Sul. Campo Largo é a cidade mais a Oeste e Campina Grande do Sul, mais ao Leste. A altitude varia de $850 \mathrm{~m}$ em Agudos do Sul até 980 m acima do nível do mar, em Bocaiuva do Sul.

A unidade fitogeográfica é caracterizada como transicional entre a Floresta Ombrófila Densa e a Floresta Ombrófila Mista, com dominância de A. angustifolia . O clima dominante da região, segundo a classificação de Köppen, é do tipo Cfb, com precipitação em todas as estações; a temperatura média do mês mais quente é inferior a $22{ }^{\circ} \mathrm{C}$. Os solos, em sua maioria, são classificados como Cambissolos álicos e apresentam-se pobres, pouco desenvolvidos, ácidos, com teores elevados de alumínio e baixa saturação de bases (BAGGIO et al., 1995). 


\subsection{Base de dados}

A base inicial de dados foi composta por 267 parcelas temporárias, instaladas em bracatingais nativos e manejados de forma tradicional na região metropolitana de Curitiba pela Embrapa - Florestas, Companhia de Energia Elétrica do Paraná - Copel, Rochadelli et al., na década de 1990, e, mais recentemente, por Machado et al., em 2006. Outras 40 parcelas foram instaladas por ocasião deste trabalho, totalizando 307 parcelas. A superfície amostral variou de $100 \mathrm{~m}^{2}$ a $400 \mathrm{~m}^{2}$ e as idades, de 3 a 18 anos. Nas unidades amostrais foram mensuradas as variáveis altura total, altura de fuste e circunferência à altura do peito de todos os indivíduos, com vara graduada e fita métrica, respectivamente.

As estimativas da biomassa (peso seco) total e da lenha de cada indivíduo dentro das parcelas foram feitas através das respectivas equações desenvolvidas por Urbano et al. (2008), as quais podem ser observadas a seguir com as respectivas estatísticas de ajuste, (1) e (2). A soma dos pesos de todos os indivíduos de cada parcela resultou na biomassa para as respectivas unidades de área. Esses valores foram extrapolados para hectare, tal como se usa nos inventários volumétricos convencionais partindo de equações de volume.

$$
\begin{aligned}
& \mathrm{BT}_{\mathrm{i}}=-11,8301+0,50917 \mathrm{dap}^{2} \\
& \mathrm{R}^{2}{ }_{\mathrm{aj}}=0,9600 \mathrm{~S}_{\mathrm{yx}} \%=21,68 \\
& \mathrm{BL}_{\mathrm{i}}=-6,6694+0,209204 \mathrm{dap}^{2}+0,012728 \mathrm{dap}^{2} \mathrm{~h} \\
& \mathrm{R}^{2}{ }_{\mathrm{aj}}=0,9590 \mathrm{~S}_{\mathrm{yx}} \%=23,47 \\
& \text { em que: }
\end{aligned}
$$

$$
\begin{aligned}
& \mathrm{BT}_{\mathrm{i}}=\text { biomassa total individual }(\mathrm{kg}) ; \\
& \mathrm{BL}_{\mathrm{i}}=\text { biomassa da lenha individual }(\mathrm{kg}) ; \\
& \mathrm{dap}=\text { diâmetro à altura do peito }(\mathrm{cm}) ; \text { e } \\
& \mathrm{h}=\text { altura total }(\mathrm{m}) .
\end{aligned}
$$

Foram também determinados a área basal e o número de árvores por hectare em cada unidade amostral, bem como idade, índice de sítio, altura total média, altura dominante, altura de fuste média, diâmetro médio e diâmetro quadrático de cada parcela.

A detecção de valores discrepantes foi realizada seguindo três critérios: quando foi possível afirmar que houve erro de medição; quando em uma parcela foram identificados valores negativos de biomassa total e da lenha; e quando foram observados valores muito superiores e inferiores nas diferentes classes de idade. Nesse procedimento, foram identificadas 35 parcelas discrepantes, as quais foram eliminadas da base de dados. Das 272 parcelas restantes, retiraram-se 17 parcelas aleatoriamente nas diferentes idades e classes de sítio, para validação dos modelos ajustados selecionados. A base de dados final, utilizada para o ajuste dos modelos estimativos da biomassa total e da lenha por unidade de área, foi, então, de 255 parcelas, para a qual se procedeu a uma análise estatística de cada variável, bem como ao cálculo do número de amostras necessárias em função da variância da população. A precisão estipulada foi de $10 \%$, com probabilidade fixada em 95\%.

\subsection{Matriz de correlação linear simples}

Uma matriz de correlação linear simples foi construída para analisar a relação das variáveis independentes com a biomassa total e da lenha, nas suas formas puras e logaritmizadas. O conjunto de variáveis independentes totalizou 85 variáveis, entre variáveis puras, inversas, combinadas, potencializadas e logaritmizadas.

\subsection{Modelos tradicionais testados}

Foram testados 10 modelos aritméticos e semilogarítmicos tradicionais obtidos na literatura, identificados de a1 até a10, destacando-se entre eles os modelos propostos por Spurr (1952), dos quais um foi adaptado da forma alemã para volume individual (a2) e o outro composto pela altura dominante (a3), muito utilizado devido à rapidez e ao baixo custo na coleta de dados em campo. Os modelos logarítmicos testados totalizaram 11 modelos, identificados de $\ln 1$ a $\ln 11$; destacou-se entre esses modelos aquele proposto por Clutter $(\ln 11)$, devido à sua ampla utilização em estudos de crescimento e produção, representando ferramenta muito útil para o manejador florestal.

Visando à correção da discrepância logarítmica para aqueles modelos logaritmizados, foi efetuado o recálculo das estatísticas de ajuste, aplicando-se o Índice de Meyer (IM).

\subsection{Desenvolvimento de equações por Stepwise}

Para cada uma das variáveis, duas equações foram desenvolvidas pelo processo Stepwise: uma com a variável dependente na forma pura e outra na forma logaritmizada. Nesse procedimento, as variáveis são

Revista Árvore, Viçosa-MG, v.38, n.1, p.53-62, 2014 
inseridas em ordem decrescente de correlação linear simples com a variável dependente e, quando não resultam em melhora significativa, são eliminadas. O nível de significância utilizado foi de $95 \%$ para a construção das equações por Stepwise.

\subsection{Seleção de equações}

A melhor equação estimativa para cada variável dependente foi selecionada comparando-se o coeficiente de determinação ajustado $\left(\mathrm{R}_{\text {aj }}^{2}\right)$, erro-padrão da estimativa percentual ( $\left.\mathrm{S}_{\mathrm{yx}} \%\right)$, teste $\mathrm{F}$ e distribuição gráfica de resíduos percentuais. Para aquelas equações em que a variável dependente foi logaritmizada ou transformada, efetuou-se o cálculo do Índice de Schlaegel (IA), compatível com o valor de coeficiente de determinação das demais equações.

\subsection{Validação das equações selecionadas}

A validação permite ao pesquisador analisar a aplicabilidade da equação estimativa desenvolvida e selecionada como de maior acurácia. Para que fossem consideradas válidas as equações selecionadas para cada caso, adotou-se o teste de aderência de Quiquadrado $\left(x^{2}\right)$, a 95\% de significância. A análise da distribuição de resíduos para as 17 parcelas retiradas da base de dados foi realizada, servindo também como critério de validação.

\section{RESULTADOS}

\subsection{Caracterização da base de dados}

Os resultados obtidos de suficiência amostral indicaram que a base de dados utilizada foi eficiente para a estimativa de todas as variáveis. O número de árvores foi responsável pelo maior número de amostras necessárias, com 205 amostras. Para todas as variáveis dependentes, o erro de amostragem relativo foi inferior a 1\%, o número de árvores foi responsável pelo menor erro, com 4,18\%; para as demais variáveis independentes, os valores de erro de amostragem relativo foram superiores a $20 \%$, com valor máximo para o diâmetro médio (40\%).

O diâmetro médio apresentou menor valor de $5,23 \mathrm{~cm}$ aos 4 anos no sítio III e maior valor $(21,59 \mathrm{~cm})$ em um povoamento de 18 anos no sítio I, com coeficiente de variação igual a 32,44\%. A altura total média teve seu valor mínimo igual a 6,91 m aos 3,5 anos no sítio

Revista Árvore, Viçosa-MG, v.38, n.1, p.53-62, 2014
III e valor máximo de 20,08 m na idade de 18 anos no sítio I, com coeficiente de variação de 19,71\%, o menor entre as variáveis.

A área basal apresentou coeficiente de variação de 34,12\%. O valor mínimo foi de 2,06 $\mathrm{m}^{2} / \mathrm{ha}$, observado em um povoamento de 14 anos no sítio III, e o valor máximo foi de 24,09 m²/ha, observado no sítio II, em um povoamento de 6 anos de idade. A menor densidade de árvores (67 árvores/ha) foi observada em um povoamento com idade de 14 anos no sítio III e a maior (6.161 árvores/ha), em um povoamento de 5 anos de idade, no sítio I. O coeficiente de variação desta variável foi de $72,72 \%$, o mais elevado entre as variáveis.

A biomassa total teve o menor valor estimado no sítio III em povoamento com 14 anos, com valor de $12.469,77 \mathrm{~kg} / \mathrm{ha}$; e maior valor (113.195,60 kg/ha) para um povoamento de 7 anos, no sítio I. O coeficiente de variação foi de 36,92\% e a amplitude, igual a $100.725,83 \mathrm{~kg} / \mathrm{ha}$. A biomassa da lenha apresentou coeficiente de variação igual a 38,63\%. O menor valor foi de $9.693,05 \mathrm{~kg} / \mathrm{ha}$, estimado para um povoamento de 14 anos no sítio III e maior valor no sítio I, em um povoamento de 10 anos, igual a $94.373,41 \mathrm{~kg} / \mathrm{ha}$, apresentando amplitude de 84.680,36 kg/ha.

\subsection{Matriz de correlação linear simples}

Na Tabela 1 é apresentada a relação das variáveis independentes que apresentaram correlação superior a 0,9 para pelo menos uma das variáveis dependentes. Foram destacadas as duas maiores correlações de cada variável.

\subsection{Equações estimativas da biomassa total}

Os coeficientes ajustados com as respectivas estatísticas de ajuste e precisão para as equações tradicionais estimativas da biomassa total são apresentados na Tabela 2. Em geral, os resultados das equações foram satisfatórios, com altos valores de $\mathrm{R}^{2}{ }_{\text {aj }}$ e baixos valores de $\mathrm{S}_{\mathrm{yx}} \%$.

No desenvolvimento da equação aritmética pelo processo Stepwise, a variável $\mathrm{G}^{2} \overline{\mathrm{d}}$ foi a primeira a ingressar, nesta sequência: $\ln G^{2} \bar{d}, N^{2}$ e $G^{2} \bar{d}$. A equação final ajustada, representada pela equação (3), apresentou $\mathrm{R}^{2}$ aj igual a 0,9932, $\mathrm{S}_{\mathrm{yx}} \%$ igual a 3,03 e valor de F igual a 9351 . $\mathrm{BT}=-48088,2975+3,9289 \mathrm{G}^{2} \overline{\mathrm{d}}+12433,1841 \ln ^{2} \overline{\mathrm{d}}-$ $-0,0013 N^{2}+99,2326 G^{2}$ 
Tabela 1 - Matriz de correlação linear simples entre as variáveis independentes mais correlacionadas com as variáveis dependentes biomassa total (BT), logaritmo neperiano da biomassa total (lnBT), biomassa da lenha (BL) e logaritmo neperiano da biomassa da lenha (lnBL).

Table 1 - Linear correlation matrix between independent variables more correlated with the dependent variables total biomass (BT), natural logarithm of total biomass ( $\operatorname{lnBT}$ ), firewood biomass (BL) and natural logarithm of firewood biomass $(\ln B L)$.

\begin{tabular}{lcccc}
\hline Variável independente & BT & $\operatorname{lnBT}$ & BL & $\operatorname{lnBL}$ \\
\hline $\mathrm{G}^{2} \overline{\mathrm{d}}$ & 0,9563 & 0,8745 & 0,9421 & 0,8656 \\
$\mathrm{G}^{2} \mathrm{~d}_{\mathrm{g}}$ & 0,9545 & 0,8719 & 0,9405 & 0,8635 \\
$\ln \mathrm{G}^{2} \overline{\mathrm{d}}$ & 0,9382 & 0,9654 & 0,9239 & 0,9546 \\
$\ln \mathrm{d}_{\mathrm{g}}$ & 0,9372 & 0,9640 & 0,9234 & 0,9537 \\
$\mathrm{G} \overline{\mathrm{h}}$ & 0,9340 & 0,9036 & 0,9707 & 0,9375 \\
$\mathrm{Gd}$ & 0,9147 & 0,8757 & 0,9308 & 0,8908 \\
$\mathrm{G} \overline{\mathrm{h}}$ & 0,9147 & 0,8408 & 0,9139 & 0,8439 \\
$\mathrm{G} \overline{\mathrm{d}}$ & 0,9134 & 0,8756 & 0,9293 & 0,8902 \\
$\mathrm{Gh}$ & 0,9014 & 0,8707 & 0,9438 & 0,9084 \\
$\ln \mathrm{dom}$ & 0,9013 & 0,9317 & 0,9300 & 0,9622 \\
$\ln \mathrm{G}^{2} \overline{\mathrm{h}}$ & 0,8824 & 0,9158 & 0,8787 & 0,9154 \\
$\ln \mathrm{Gh}_{\mathrm{dom}}$ & 0,8725 & 0,9039 & 0,9061 & 0,9372 \\
$\ln \mathrm{G}^{2} h_{\text {dom }}$ & 0,8680 & 0,9019 & 0,8676 & 0,9036 \\
$\ln \bar{h}^{2}$ & 0,8308 & 0,8534 & 0,9051 & 0,9270 \\
$\mathrm{Gh}^{2}$ & 0,8228 & 0,7934 & 0,9103 & 0,8672 \\
\hline
\end{tabular}

$\mathrm{G}=$ Área basal por hectare $\left(\mathrm{m}^{2} / \mathrm{ha}\right) ; \overline{\mathrm{h}}=$ Altura total média $(\mathrm{m}) ; \mathrm{h}_{\mathrm{dom}}=$ Altura dominante $(\mathrm{m}) ; \overline{\mathrm{d}}=$ Diâmetro à altura do peito médio (cm); $\mathrm{d}_{\mathrm{g}}=$ Diâmetro médio quadrático $(\mathrm{cm})$; e $\mathrm{ln}=$ Logaritmo neperiano.

em que:

$\mathrm{BT}=$ biomassa total $(\mathrm{kg} / \mathrm{ha})$;

$\mathrm{G}=$ área basal (G/ha);

$\overline{\mathrm{d}}=$ diâmetro médio à altura do peito $(\mathrm{cm})$; e

$\mathrm{N}$ = número de indivíduos (N/ha).

No caso da equação logarítmica desenvolvida por Stepwise, o valor $\mathrm{R}_{\text {aj }}^{2}$ para a equação final representado pela equação (4) foi igual a 0,9921, com S $\mathrm{yx}_{\mathrm{x}}$ \% de 3,28 e valor de F igual a 8213. O Índice de Meyer calculado para essa equação foi de 1,0011. As variáveis independentes que compuseram a equação final ajustada foram $\ln G^{2} \overline{\mathrm{d}}, \mathrm{N}^{2}$, GN e $G \overline{\mathrm{d}}^{2}$.

$\operatorname{lnBT}=7,0953+0.5397 \operatorname{lnG}^{2}-0,000000034 N^{2}+$

$+0,000005718 \mathrm{GN}-0,000039315 \mathrm{G}^{2}$

em que:

$\operatorname{lnBT}=$ logaritmo neperiano da biomassa da lenha $(\mathrm{kg} / \mathrm{ha})$;

$\mathrm{G}=$ área basal (m²/ha); e

$\overline{\mathrm{d}}=$ diâmetro médio à altura do peito $(\mathrm{cm})$.

Na Figura 1, pode ser observada a distribuição de resíduos para as equações a6 e $\ln 11$, selecionadas entre as aritméticas como as mais acuradas, e para as equações semilogarítmica e logarítmica, desenvolvidas por Stepwise. A distribuição de resíduos para as quatro equações foi homogênea, independente e pouco dispersa ao longo da linha estimativa, o que dificultou a comparação gráfica delas. As demais equações tradicionais testadas apresentaram superestimativas para valores inferiores a $50.000 \mathrm{~kg} / \mathrm{ha}$ de biomassa total.

A equação $\ln 11$ foi selecionada como a mais acurada entre as tradicionais, pela superioridade que apresentou nas estatísticas de ajuste e precisão, sendo graficamente muito semelhante à equação a6. Entre as equações de Stepwise, graficamente também não se observou diferença, sendo a semilogarítmica considerada melhor por causa do melhor resultado encontrado para $\mathrm{R}^{2}{ }_{\text {aj' }}$, $\mathrm{S}_{\mathrm{yx}} \%$ e valor de $\mathrm{F}$.

As equações selecionadas foram validadas pelo teste de aderência de Qui-quadrado, com valores de $x^{2}$ calculado iguais a 1,2145 e 0,0374 , respectivamente para a equação $\ln 11$ e Stepwise. O valor tabelado de $x^{2}$ foi de 26,30. A dispersão de resíduos da biomassa total para as 17 unidades amostrais utilizadas na validação das equações apresentou-se homogênea, independente e pouco dispersa ao longo da linha estimativa de ambas

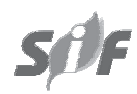

Revista Árvore, Viçosa-MG, v.38, n.1, p.53-62, 2014 
Tabela 2 - Equações tradicionais ajustadas com as respectivas estatísticas de ajuste e precisão, para estimativa da biomassa total (kg/ha).

Table 2 - Traditional adjusted equations with respective statistics for total biomass estimative ( $\mathrm{kg} / \mathrm{ha}$ ).

\begin{tabular}{|c|c|c|c|c|c|c|}
\hline Modelo & Ajuste & Autor & $\mathrm{R}^{2} \mathrm{aj}$ & Syx\% & $\mathrm{F}$ & IM \\
\hline a 1 & $\mathrm{BT}=4994,9193+4408,8851 \mathrm{G}$ & Spurr (1952) & 0,7079 & 19,95 & 617 & - \\
\hline a2 & $\mathrm{BT}=4213,1026+358,5025 \mathrm{G} \overline{\mathrm{h}}$ & Spurr (1952) & 0,8719 & 13,21 & 1730 & - \\
\hline a3 & $\mathrm{BT}=7779,2054+301,4810 \mathrm{Gh}_{\mathrm{dom}}$ & Spurr (1952) & 0,8117 & 16,02 & 1096 & - \\
\hline a4 & $\mathrm{BT}=27016,4826+15,6442 \mathrm{G}^{2} \overline{\mathrm{h}}$ & Rosot (1989) & 0,8359 & 14,95 & 1295 & - \\
\hline a5 & $\mathrm{BT}=28313,3560+13,4634 \mathrm{G}^{2} \mathrm{~h}_{\mathrm{dom}}$ & Silva (1979) & 0,7964 & 16,66 & 995 & - \\
\hline a6* & $\mathrm{BT}=6,5512+\mathrm{G}^{0,9306}+\overline{\mathrm{h}} 0,8315$ & Ung e Ouellet (1991) & 0,8783 & 12,88 & 893 & 1,0110 \\
\hline a 7 & $\mathrm{BT}=6,9281+\mathrm{G}^{0,9003}+\mathrm{h}_{\mathrm{dom}} 0,6839$ & Ung e Ouellet (1991) & 0,8314 & 15,01 & 610 & 1,0147 \\
\hline a8 & $\mathrm{BT}=-163659,4503+44882,1866 \ln \mathrm{G} \overline{\mathrm{h}}$ & Figueiredo (1983) & 0,8117 & 16,02 & 1096 & - \\
\hline a9 & $\mathrm{BT}=-153793,9357+42036,2611 \ln \mathrm{Gh}_{\mathrm{dom}}$ & Figueiredo (1983) & 0,7603 & 18,07 & 807 & - \\
\hline a10 & $\mathrm{BT}=-113376,4599+23284,6515 \ln \mathrm{G}^{2} \overline{\mathrm{h}}$ & Figueiredo (1983) & 0,7777 & 17,41 & 890 & - \\
\hline $\ln 1$ & $\ln B T=8,7798+0,8728 \ln G$ & Spurr (1952) & 0,7093 & 20,00 & 578 & 1,0247 \\
\hline $\ln 2$ & $\ln \mathrm{BT}=6,3831+0,9140 \ln \mathrm{G} \overline{\mathrm{h}}$ & Spurr (1952) & 0,8807 & 13,22 & 1712 & 1,0111 \\
\hline $\ln 3$ & $\operatorname{lnBT}=6,55747+0,8580 \ln \mathrm{Gh}_{\mathrm{dom}}$ & Spurr (1952) & 0,8123 & 16,00 & 1116 & 1,0155 \\
\hline $\ln 4$ & $\operatorname{lnBT}=7,3934+0,4761 \ln G^{2} \overline{\mathrm{h}}$ & Figueiredo (1983) & 0,8564 & 13,99 & 1406 & 1,0136 \\
\hline $\ln 5$ & $\operatorname{lnBT}=7,4574+0,4612 \operatorname{lnG}{ }^{2} h_{d o m}$ & Spurr (1952) & 0,8252 & 15,44 & 1125 & 1,0158 \\
\hline $\ln 6$ & $\operatorname{lnBT}=6,3905+1,1730 \ln G \bar{h}-0,2554 \operatorname{lnGh} \mathrm{dom}_{1}$ & Ung e Ouellet (1991) & 0,8738 & 13,11 & 874 & 1,0109 \\
\hline $\ln 7$ & $\operatorname{lnBT}=9,2694+0,1680 \ln G N$ & Rosot (1989) & 0,1664 & 33,71 & 74 & 1,0668 \\
\hline $\ln 8$ & $\operatorname{lnBT}=8,9879+0,1580 \ln G^{2} N$ & Scolforo (1997) & 0,2820 & 31,28 & 123 & 1,0566 \\
\hline $\ln 9$ & $\ln \mathrm{BT}=10,1246+0,1024 \ln \overline{\mathrm{d}}^{2} \overline{\mathrm{h}}$ & Rosot (1989) & 0,0362 & 36,24 & 13 & 1,0838 \\
\hline $\ln 10$ & $\operatorname{lnBT}=7,6907+0,3364 \ln N \bar{d}$ & Scolforo (1997) & 0,1657 & 33,72 & 74 & 1,0669 \\
\hline $\ln 11^{*}$ & $\operatorname{lnBT}=8,6367+0,0201 S-3,7531 \mathrm{I}-1+1,0203 \ln G$ & Clutter et al. (1983) & 0,8808 & 12,75 & 621 & 1,0098 \\
\hline
\end{tabular}

$\mathrm{R}_{\mathrm{aj}}^{2}=$ Coeficiente de determinação ajustado; $\mathrm{S}_{\mathrm{yx}} \%$ = Erro-padrão da estimativa percentual; F = Teste F; IM = Índice de Meyer; G = Área basal $\left(\mathrm{m}^{2} / \mathrm{ha}\right) ; \overline{\mathrm{h}}=$ Altura total média $(\mathrm{m}) ; \mathrm{h}_{\mathrm{dom}}=$ Altura dominante $(\mathrm{m}) ; \overline{\mathrm{d}}=$ Diâmetro médio à altura do peito $(\mathrm{cm}) ; \mathrm{N}=\mathrm{Número} \mathrm{de}$ indivíduos (N/ha); S = Índice de sítio; I = idade (anos); ln = logaritmo neperiano; e * = Equações selecionadas de melhor ajuste para cada caso.

as equações, atestando, assim, a aderência desses resíduos para as estimativas.

\subsection{Equações estimativas da biomassa da lenha}

Em geral, os resultados do ajuste dos modelos tradicionais para estimativa da biomassa da lenha foram satisfatórios. Elevados valores de $\mathrm{R}_{\text {aj }}^{2}$ e baixos valores de $\mathrm{S}_{\mathrm{yx}} \%$ foram observados tanto nas equações aritméticas e semilogarítmicas quanto nas equações logarítmicas, salvo exceções, como pode ser observado na Tabela 3.

No desenvolvimento da equação aritmética pelo processo Stepwise, a variável independente que primeiro ingressou foi $G \bar{h}$, seguida por $N \bar{h}$ e $G \bar{h}^{2}$. A equação final ajustada, representada pela equação (5), apresentou resultado satisfatório, com $\mathrm{R}^{2}$ aj igual a $0,9965, \mathrm{~S}_{\mathrm{yx}} \%$ de 2,27 e valor de F igual a 24449.

$\mathrm{BL}=-439,4829+545,2247 \mathrm{G} \overline{\mathrm{h}}-0,5901 \mathrm{~N} \overline{\mathrm{h}}-$

$-2,2338 G \bar{h}^{2}$ em que:

$\mathrm{BL}=$ biomassa da lenha $(\mathrm{kg} / \mathrm{ha})$;

$\mathrm{G}=$ área basal (m²/ha);

$\overline{\mathrm{h}}=$ altura total média (m); e

$\mathrm{N}=$ número de indivíduos (N/ha).

A equação logarítmica final, desenvolvida pelo processo Stepwise para a estimativa da biomassa da lenha, é representada pela equação 6; para essa equação, o valor de $\mathrm{R}^{2}$ aj foi igual a $0,9934, \mathrm{~S}_{\mathrm{yx}} \%$ de 3,12 e valor de F de 13100. O Índice de Meyer calculado foi de 1,0006. A variável combinada $\ln G \bar{h}$ foi a primeira variável incorporada, seguida por $\overline{\mathrm{d}}^{-1}$ e $\ln \overline{\mathrm{d}} \overline{\mathrm{h}}$.

$\operatorname{lnBL}=9,4694+1,0255 \ln G \bar{h}-11,0636 \bar{d}^{-1}-$

- 0,5657ln $\bar{d} \bar{h}$

em que:

$\operatorname{lnBL}=$ logaritmo neperiano da biomassa da lenha (kg/ha); 
Tabela 3 - Equações tradicionais ajustadas com as respectivas estatísticas de ajuste e precisão, para estimativa da biomassa da lenha (kg/ha).

Table 3 - Traditional adjusted equations with respective statistical of fitting and precision for firewood biomass estimate $(\mathrm{kg} / \mathrm{ha})$.

\begin{tabular}{|c|c|c|c|c|c|c|}
\hline Modelo & Ajuste & Autor & $R^{2} a j$ & Syx\% & $\mathrm{F}$ & $\mathrm{IM}$ \\
\hline a 1 & $\mathrm{BL}=4261,2295+3487,7828 \mathrm{G}$ & Spurr (1952) & 0,6372 & 23,27 & 447 & - \\
\hline a2 & $\mathrm{BL}=-265,8174+310,5602 \mathrm{G} \overline{\mathrm{h}}$ & Spurr (1952) & 0,9420 & 9,30 & 4128 & - \\
\hline a3 & $\mathrm{BL}=2510,2625+263,1139 \mathrm{Gh}_{\mathrm{dom}}$ & Spurr (1952) & 0,8902 & 12,80 & 2061 & - \\
\hline a4 & $\mathrm{BL}=20464,2646+13,0287 \mathrm{G}^{2} \overline{\mathrm{h}}$ & Rosot (1989) & 0,8345 & 15,72 & 1282 & - \\
\hline a5 & $\mathrm{BL}=21408,5463+11,2781 \mathrm{G}^{2} \mathrm{~h}_{\mathrm{dom}}$ & Silva (1979) & 0,8044 & 17,08 & 1046 & - \\
\hline a6* & $\mathrm{BL}=5,4759+\mathrm{G}^{0,9567}+\overline{\mathrm{h}}^{1,1411}$ & Ung e Ouellet (1991) & 0,9410 & 9,38 & 2017 & 1,0063 \\
\hline a 7 & $\mathrm{BBL}=5,8633+\mathrm{G}^{0,9171}+\mathrm{h}_{\mathrm{dom}}^{0,9866}$ & Ung e Ouellet (1991) & 0,8884 & 12,91 & 1007 & 1,0112 \\
\hline a 8 & $\mathrm{BL}=-144317,5229+38600,1642 \ln \mathrm{G} \overline{\mathrm{h}}$ & Figueiredo (1983) & 0,8643 & 14,23 & 1619 & - \\
\hline a9 & $\mathrm{BL}=-137013,8700+36388,9930 \ln \mathrm{Gh}_{\mathrm{dom}}$ & Figueiredo (1983) & 0,8203 & 16,37 & 1161 & - \\
\hline a10 & $\mathrm{BL}=-95988,5800+19327,4771 \operatorname{lnG}^{2} \overline{\mathrm{h}}$ & Figueiredo (1983) & 0,7712 & 18,48 & 857 & - \\
\hline $\ln 1$ & $\operatorname{lnBL}=8,5344+0,8773 \ln G$ & Spurr (1952) & 0,6372 & 23,27 & 431 & 1,0323 \\
\hline $\ln 2$ & $\operatorname{lnBL}=5,7886+0,9875 \ln G \bar{h}$ & Spurr (1952) & 0,9416 & 9,33 & 3958 & 1,0068 \\
\hline $\ln 3$ & $\operatorname{lnBL}=5,9768+0,9307 \operatorname{lnGh} \mathrm{dom}$ & Spurr (1952) & 0,8900 & 12,81 & 2020 & 1,0112 \\
\hline $\ln 4$ & $\operatorname{lnBL}=7,0002+0,4979 \ln G^{2} \overline{\mathrm{h}}$ & Figueiredo (1983) & 0,8542 & 14,75 & 1371 & 1,0150 \\
\hline $\ln 5$ & $\operatorname{lnBL}=7,0589+0,4834 \ln \mathrm{G}^{2} \mathrm{~h}_{\mathrm{dom}}$ & Spurr (1952) & 0,8309 & 15,89 & 1157 & 1,0170 \\
\hline $\ln 6$ & $\operatorname{lnBL}=5,7936+1,1663 \ln G \bar{h}-0,1763 \ln G h_{d o m}$ & Ung e Ouellet (1991) & 0,9414 & 9,35 & 1975 & 1,0067 \\
\hline $\ln 7$ & $\ln B L=9,1487+0,1560 \ln G N$ & Rosot (1989) & 0,1191 & 36,26 & 56 & 1,0781 \\
\hline $\ln 8$ & $\ln B L=8,8336+0,1512 \ln G^{2} N$ & Scolforo (1997) & 0,2221 & 34,07 & 96 & 1,0676 \\
\hline $\ln 9$ & $\operatorname{lnBL}=9,5590+0,1484 \ln \bar{d}^{2} \overline{\mathrm{h}}$ & Rosot (1989) & 0,0724 & 37,21 & 26 & 1,0884 \\
\hline $\ln 10$ & $\ln B L=7,6896+0,3116 \ln N \bar{h}$ & Scolforo (1997) & 0,1177 & 36,29 & 55 & 1,0783 \\
\hline $\ln 11^{*}$ & $\operatorname{lnBL}=8,1770+0,0422 \mathrm{~S}-4,4911 \mathrm{I}-1+1,0321 \ln \mathrm{g}$ & Clutter et al. (1983) & 0,9093 & 11,64 & 872 & 1,0083 \\
\hline
\end{tabular}

$\mathrm{R}^{2}$ = Coeficiente de determinação ajustado; S \% = Erro-padrão da estimativa percentual; F = Teste F; IM = Índice de Meyer; G = Área basal $\left(\mathrm{m}^{2} / \mathrm{ha}\right) ; \overline{\mathrm{h}}=$ Altura total média $(\mathrm{m}) ; \mathrm{h}$, Altura dominante $(\mathrm{m}) ; \overline{\mathrm{d}}=$ Diâmetro médio à altura do peito $(\mathrm{cm}) ; \mathrm{N}=\mathrm{Nú}$ mero de indivíduos (N/ha); $\mathrm{S}=$ Indice de sítio; I = idade (anos); ln = logaritmo neperiano; $\mathrm{e}^{*}=$ Equações selecionadas de melhor ajuste para cada caso.

$\mathrm{G}=$ área basal (m²/ha);

$\overline{\mathrm{h}}=$ altura total média $(\mathrm{m})$; e

$\overline{\mathrm{d}}=$ diâmetro médio à altura do peito $(\mathrm{cm})$.

Na Figura 2 pode ser observada a distribuição percentual de resíduos da biomassa da lenha para as equações a2 e $\ln 2$, selecionadas entre as equações tradicionais aritméticas e logarítmicas, e para as equações desenvolvidas por Stepwise, para ambos os casos.

Da mesma forma que na biomassa total, não foi possível identificar graficamente diferenças entre as equações selecionadas, e todas apresentaram homogeneidade, independência e baixa dispersão de resíduos ao longo da linha estimativa. Foram consideradas mais acuradas, então, aquelas equações que apresentaram melhores estatísticas de ajuste e precisão, resultando nas equações a2 e aritmética de Stepwise.

Ambas as equações foram consideradas válidas pelo teste de Qui-quadrado, com valores de $x^{2}$ iguais a 0,6616 e 0,0312, respectivamente para a equação a2 e Stepwise. O valor tabelado de $x^{2}$ foi igual a 26,30. A análise gráfica das 17 parcelas separadas da base de dados comprovou também a validade dessas equações, em que a distribuição de resíduos foi homogênea, independente e pouco dispersa ao longo da linha estimativa.

\section{DISCUSSÃO}

\subsection{Análise da base de dados}

Diante dos resultados da caracterização da base de dados, é possível afirmar que essa caracterização se apresentou sólida, representativa das diferentes combinações de idades e sítios existentes na região metropolitana de Curitiba. Destaca-se, porém, o caso da área basal, que apresentou o maior valor para um povoamento de 6 anos no sítio II e que foi justificado pelo elevado número de indivíduos e diâmetro médio constatados nas unidades amostrais dessa combinação.

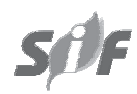

Revista Árvore, Viçosa-MG, v.38, n.1, p.53-62, 2014 
A matriz de correlação linear simples proporcionou resultados importantes para o processo de seleção das equações, permitindo a detecção rápida de quais variáveis independentes foram altamente correlacionadas com as dependentes. Estudos relacionados à estimativa do volume por unidade de área têm utilizado essa ferramenta para a mesma finalidade, como pode ser observado nos trabalhos de Oliveira et al. (2005) e Machado et al. (2008).

A maior correlação com a combinação $G$ foi observada apenas para a biomassa da lenha; para a biomassa total, a melhor relação foi com a combinação $\mathrm{G}^{2}$. Esse resultado corrobora o do trabalho apresentado por Urbano (2008), que destacou a maior relação da biomassa total individual com o diâmetro à altura do peito.

\subsection{Seleção de equações}

Para a biomassa total, como mencionado anteriormente, as equações selecionadas foram a equação logarítmica de Clutter $(\ln 11)$ e a equação aritmética desenvolvida por Stepwise.

а6

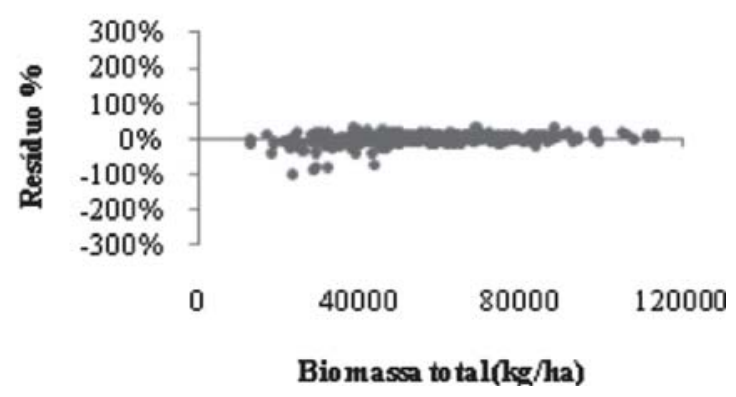

Stepwise semilogarítmico

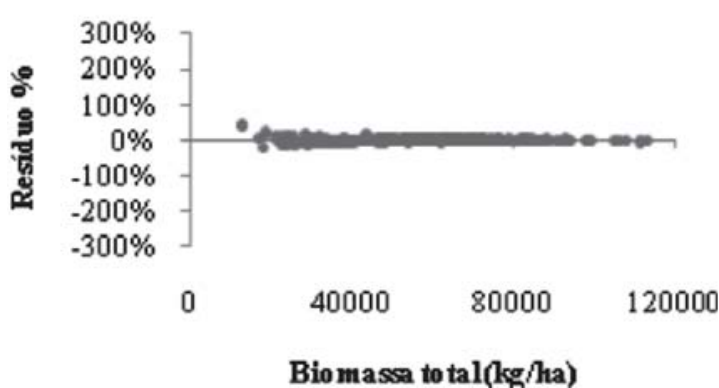

O resultado da equação de Clutter merece destaque, por ser aplicado em estudos de crescimento e produção e pelo fato de envolver as variáveis idade, sítio e área basal, as quais são de fácil obtenção em campo. Sobre as equações desenvolvidas por Stepwise, destaca-se a menor correlação linear simples das variáveis que envolveram a altura, induzindo que estas não fossem incluídas nas equações. Esse resultado difere dos encontrados por Silva (1979), Figueiredo Filho (1982), Rosot (1989), Oliveira et al. (2005) e Machado et al. (2008), nos quais as equações de Stepwise para a estimativa do volume por unidade de área envolveram sempre variáveis combinadas à altura.

Urbano et al. (2008), quando desenvolveram equações para a biomassa total individual da bracatinga, também selecionaram e indicaram para uso a equação que envolveu apenas o diâmetro. A não inclusão da variável altura nas equações ajustadas neste trabalho é explicada pelo fato de a biomassa total envolver todos os compartimentos das árvores e não apenas o compartimento fuste, como ocorre nas estimativas do volume.

$\ln 11$

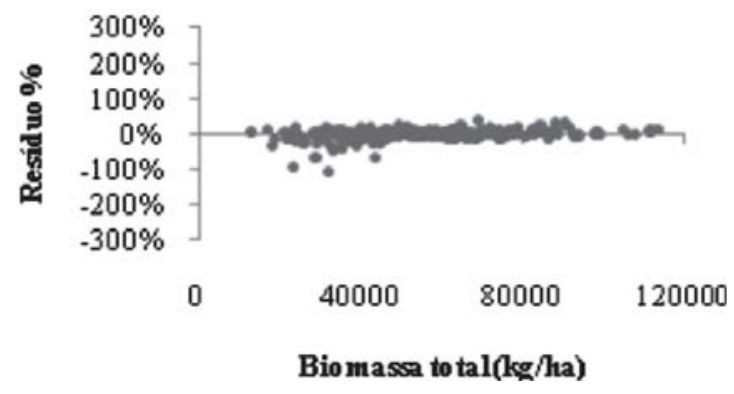

Stepwise logaritmico

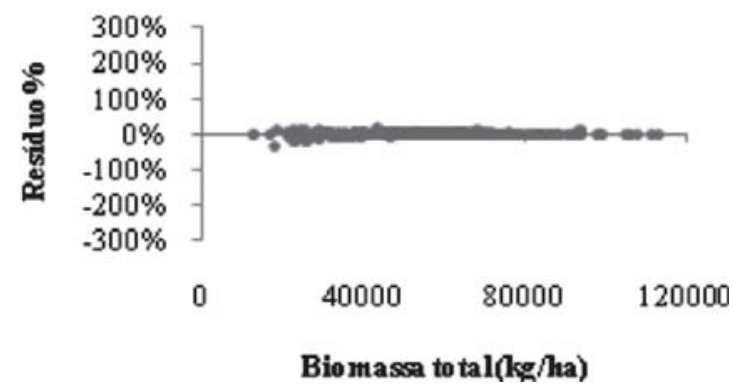

Figura 1 - Dispersão de resíduos e porcentual da biomassa total (kg/ha), para as equações a6 e ln11 e para as equações semilogarítmica e logarítmica desenvolvidas por Stepwise.

Figure 1 - Residual dispersion for total biomass ( $\mathrm{kg} / \mathrm{ha}$ ) for equations a6, ln11 and arithmetic and logarithmic equations developed by Stepwise.

Revista Árvore, Viçosa-MG, v.38, n.1, p.53-62, 2014 
a2

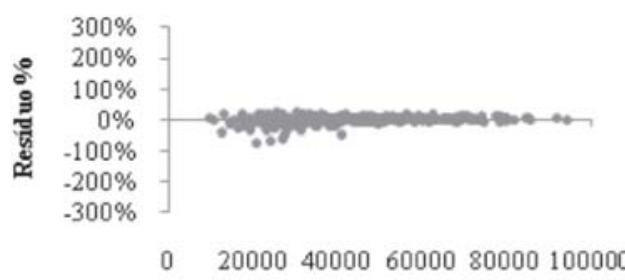

Biomassa lenha $(\mathrm{kg} / \mathrm{ha})$

Stepwise aritmético

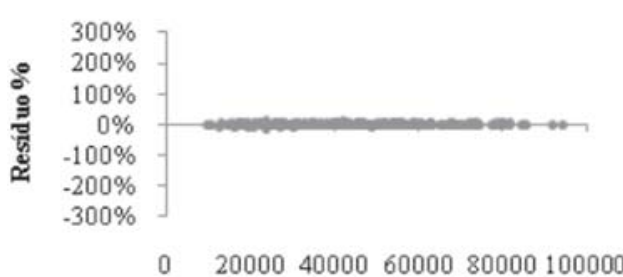

Biomassa da lenha (kg/ha) $\ln 2$

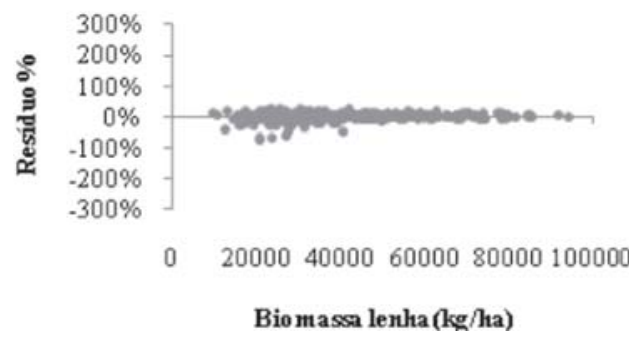

Stepwise logaritmico

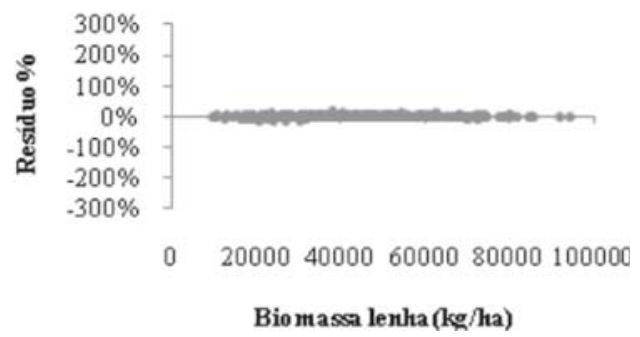

Figura 2 - Dispersão de resíduos e porcentual da biomassa da lenha (kg/ha), para as equações a2 e ln2 e para as equações aritmética e logarítmica desenvolvidas pelo processo Stepwise.

Figure 2 - Residual dispersion of firewood biomass ( $\mathrm{kg} / \mathrm{ha}$ ) for equations a2, In2 and arithmetic and logarithmic equations developed by Stepwise.

Os resultados do ajuste das equações tradicionais e do processo Stepwise para estimativa da biomassa da lenha seguiram a tendência da matriz de correlação, sendo $\mathrm{G}$ a primeira variável a ser incluída na equação. A equação tradicional selecionada foi a equação a2, proposta por Spurr e denominada como o modelo da variável combinada. Pelo processo Stepwise, a equação selecionada foi a aritmética.

O modelo proposto por Clutter, ajustado para a estimativa da biomassa da lenha, apresentou $\mathrm{R}^{2}$ aj superior a 0,90 e $\mathrm{S}_{\mathrm{yx}} \%$ de 11,64. Resultado esse que merece destaque, uma vez que possibilita a estimativa da biomassa da lenha a partir da idade e do sítio, sendo necessária apenas a determinação da área basal nas diferentes idades.

Neste trabalho, os modelos logarítmicos não apresentaram resultados superiores aos aritméticos, contrariando os resultados encontrados por Silva (1979), Figueiredo Filho (1982), Rosot (1989), Oliveira et al. (2005) e Machado et al. (2008), os quais destacaram que sempre os modelos logarítmicos apresentaram maior acurácia que os modelos aritméticos. Sobre esse aspecto,
Machado et al. (2002) citaram que é recomendável testar vários modelos, identificando por meio de análises estatísticas o melhor para cada caso.

\section{CONCLUSÕES}

As equações estimativas por unidade de área apresentaram-se muito eficientes e acuradas para estimar a biomassa total e da lenha dos bracatingais da região metropolitana de Curitiba, nas diferentes idades.

A equação de Clutter ajustada apresentou elevada acurácia para ambas as variáveis dependentes estimadas e representa alternativa importante durante o processo de comercialização dos bracatingais, permitindo a utilização do método de Bitterlich. Esse método, por sua vez, permite a redução considerável de tempo e custos no inventário florestal.

\section{AGRADECIMENTOS}

À EMBRAPA-FLORESTAS, COPELe ao pesquisador Rochadelli, pela disponibilização de dados; e ao CNPq, pelo auxílio financeiro durante a realização dos trabalhos de campo.

Revista Árvore, Viçosa-MG, v.38, n.1, p.53-62, 2014 


\section{REFERÊNCIAS}

BAGGIO, A. J.; CARPANEZZI, A. A.; SANMIGUEL, A. A. Equações para a estimativa de peso da biomassa aérea de bracanga (Mimosa scabrella Benth.) na idade de corte. Boletim de Pesquisa Florestal, n.30/31, p.37-49, 1995.

CARPANEZZI, A. A.; CARPANEZZI, O. T. B.; BAGGIO, A. J. Manejo de bracatingais. In: GAlVÃO, A. P. M., (Org.). Tópicos em manejo florestal sustentável. Curitiba: Embrapa/Cnpf, 1997. v. único. p.157-163.

FIGUEIREDO FILHO, A.; MACHADO, S. A.; ROSOT, N. C. Estimativas volumétricas por Unidade de Área em Florestas Naturais. Silvicultura Em São Paulo, v. 16, p. 491504, 1982.

LAURENT, J. M. E. Sistema agroflorestal da barcatinga na região metropolitana de Curitiba: contexto sócio-economico. Curitiba: FAO - Projeto GOP/BRA/025/FRA, 1990. 50p. (Série Estudos Florestais, 3).

MACHADO, S. A.; CONCEIÇÃO, M. B.; FIGUEIREDO FILHO, A. Modelagem do volume individual para diferentes idades e regimes de desbaste em plantações de Pinus oocarpa. Guarapuava. Revista Ciências Exatas e Naturais, v.4, n.2, p.41-50, 2002.
MACHADO, S. A.; PROFUMO AGUIAR, L.; FIGUEIREDO FILHO, A.; KOEHLER, H. S. Modelagem do volume do povoamento para mimosa scabrella benth. na região metropolitana de Curitiba. Revista Árvore, v.32, n.3, p.465478, 2008.

OLIVEIRA, M. L. R. et al. Equações de volume de povoamento para fragmentos florestais naturais do Município de Viçosa, Minas Gerais. Revista Árvore, v.29, n.2, p.213-225, 2005.

ROSOT, M. A. D. Estudo comparativo de métodos para a avaliação volumétrica por unidade de área em um povoamento de Pinus taeda L. 1989. $163 f$. Dissertação (Mestrado em Engenharia Florestal) Setor de Ciências Agrárias, Universidade Federal do Paraná, Curitiba, 1989.

SILVA, J. A. Estimativa do volume por hectare e sua composição em povoamentos de Eucalyptus spp. nos cerrados em Minas Gerais e Mato Grosso do Sul. 1979. 85f. Dissertação (Mestrado em Engenharia Florestal) - Setor de Ciências Agrárias, Universidade Federal do Paraná, Curitiba, 1979.

URBANO, E. et al. Modelagem da biomassa aérea em Bracatingais Nativos da Região Metropolitana de Curitiba. Revista Floresta, v.38, n.2, p.361372, 2008. 